\title{
The role of precise imaging with intravascular ultrasound in coronary and peripheral interventions
}

This article was published in the following Dove Press journal: Vascular Health and Risk Management

\section{Nicolas W Shammas \\ Qais Radaideh \\ W John Shammas \\ Ghassan E Daher \\ Rayan Jo Rachwan \\ Yazan Radaideh}

Midwest Cardiovascular Research Foundation, Davenport, IA, USA
Correspondence: Nicolas W Shammas Midwest Cardiovascular Research Foundation, 1622 E Lombard Street, Davenport, IA 52803, USA

Emailshammas@mchsi.com

\begin{abstract}
Angiography remains a widely utilized imaging modality during vascular procedures. Angiography, however, has its limitations by underestimating the true vessel size, plaque morphology, presence of calcium and thrombus, plaque vulnerability, true lesion length, stent expansion and apposition, residual narrowing post intervention and the presence or absence of dissections. Intravascular ultrasound (IVUS) has emerged as an important adjunctive modality to angiography. IVUS offers precise imaging of the vessel size, plaque morphology and the presence of dissections and guides interventional procedures including stent sizing, assessing residual narrowing and stent apposition and expansion. IVUS-guided treatment has shown to yield superior outcomes when compared to angiography-only guided therapy. The cost-effectiveness of the routine use of IVUS during vascular procedures needs to be further studied.
\end{abstract}

Keywords: intravascular ultrasound, vessel size, peripheral artery disease, coronary artery disease, dissections, thrombus, stent apposition, plaque morphology

\section{Introduction}

In the early part of the 20th century, cardiac catheterization and cine angiography revolutionized the field of cardiovascular medicine and paved the way for a multitude of therapeutic interventions, most importantly bypass surgery and endovascular interventions. ${ }^{1}$ The ability to visualize and cannulate vessels also led to innovations in assessing the functional significance and morphology of lesions.

Despite significant improvements in image acquisition using angiographic techniques, cine angiography continued to have important limitations in assessing several variables including the true vessel size, plaque morphology, presence of calcium and thrombus, plaque vulnerability, true lesion length, the physiologic importance of a lesion, stent expansion and apposition, residual narrowing post intervention and the presence or absence of dissections. As of today, angiography remains the main imaging modality used worldwide for vascular imaging. Recently, several adjunctive tools to angiography have been introduced that enhanced vessel and plaque visualization and allowed physiologic assessment of lesions. These included intravascular ultrasound (IVUS), optical coherence tomography (OCT), near-infrared spectroscopy (NIRS), and flow wires with or without adenosine administration. In this review, we will be discussing IVUS and its role in vascular imaging. 


\section{Intravascular ultrasound}

IVUS during angiographic procedures offers valuable information about plaque morphology (fibrous, fibrofatty, calcified, necrotic), true vessel size and lumen area, extent and depth of calcium and dissections. IVUS was also shown to optimize stent deployment with improved outcomes when compared to angiography alone. ${ }^{2}$

IVUS catheters are currently manufactured by two major companies, Boston scientific and Philips after its acquisition of Volcano corporation in 2015. The catheter sizes range from 2-4 Fr and can be easily guided through a 5-6 Fr femoral sheath. Larger IVUS catheters are also used for larger peripheral vessel applications and require $8 \mathrm{Fr}$ sheaths (Table 1). IVUS uses a piezoelectric transducer located at the tip of the catheter that generates sound waves when electrically stimulated. These waves propagate into different tissues and thus produce a reflection image based on the acoustic properties of that tissue. The transducer is found in two different designs: 1) the mechanical single rotating device and 2) the electronic-phased array device (several non-rotating transducers present at the tip of the catheter and activated sequentially). Early IVUS imaging was in gray-scale and plaque morphology was classified based on visual appearance by comparing the echogenicity of the plaque to its surrounding adventitia. This has been possible with the use of $20-40 \mathrm{MHz}$ transducers. The plaques were classified into four groups: 1) soft plaque, 2) fibrous plaque, 3) calcified plaque and 4) mixed plaques. ${ }^{3}$ Virtual histology-IVUS (VHIVUS) was later developed to better assess and characterize the histological composition of the vessel plaque via the analysis of an additional low radiofrequency (RF) content. This RF signal, in addition to the gray-scale signal, is processed using an autoregressive model and matched to colorcoded histological databases to classify plaques according to their morphological composition. Using VH-IVUS plaques is classified as 1) fibrous tissue, 2) fibrofatty tissue, 3) necrotic core and 4) dense calcium. Two new RF-based IVUS modalities have evolved and include iMAP-IVUS and integrated backscattered IVUS (IB-IVUS) that use more sophisticated software algorithms and thus better tissue characterization. ${ }^{4}$ In this systematic review of the literature, we selected research studies with relevant clinical applications to the practicing endovascular operators.

\section{Plaque morphology}

The PROSPECT trial (Providing Regional Observations to Study Predictors of Events in the Coronary Tree) that examined the predictors of plaque rupture in patients with acute coronary syndrome showed that plaque burden, minimal luminal area and plaque composition as seen by IVUS predicted cardiovascular events. ${ }^{5}$ This was a prospective study that included 697 patients with acute coronary syndrome who have undergone coronary angiography followed by gray scale and RF IVUS evaluation. The primary endpoint included a composite of major cardiovascular events; cardiac arrest, myocardial infarction, re-hospitalization or death due to cardiac cause with a mean follow-up time of 3 years. Results showed that the rate of major cardiovascular events was $20.4 \%$ of which $11.6 \%$ were attributed to non-culprit lesions, with a mean diameter stenosis of $32.3 \pm 20.6 \%$, previously considered to be angiographically mild at initial presentation. These lesions were further characterized to have a high plaque burden (>70\%) (HR, 5.03; 95\% CI, 2.51-10.11; $P<0.001)$, minimal luminal area $\left(<4 \mathrm{~mm}^{2}\right)$ (HR, 3.21; 95\% CI, 1.61-6.42; $P=0.001$ ) or thin-cap fibroatheroma (HR, 3.35; 95\% CI, 1.77 $6.36 ; P<0.001)$ as detected by gray scale and RF-IVUS, respectively. Although this study was done in coronary arteries, it sheds light on the importance of plaque characterization with IVUS and the need to go beyond angiographic imaging in other vascular beds.

Other imaging modalities besides IVUS to characterize plaque morphology include multidetector $\mathrm{CT}$ angiography (MDCT), magnetic resonance imaging (MRI), nuclear imaging modalities, OCT and NIRS. The reader

Table I Intravascular ultrasound (IVUS) catheters for peripheral vascular disease

\begin{tabular}{|l|l|l|l|l|l|}
\hline Name of IVUS & Manufacturer & Frequency (MHZ) & $\begin{array}{l}\text { Max imaging } \\
\text { diameter (MM) }\end{array}$ & $\begin{array}{l}\text { Guide wire } \\
\text { compatibility }\end{array}$ & Images \\
\hline VISION PV 0.014 & Philips & 20 & 20 & 0.014 " & Gray-scale, VH, ChromaFlo \\
VISION PV 0.018 & Philips & - & 24 & 0.018 " & Gray-scale, ChromaFlo \\
VISION PV 0.035 & Philips & 10 & 60 & 0.035 " & Gray-scale \\
VISION PV 0.14P RX & Philips & 20 & 20 & 0.014 " & Gray-scale, VH, ChromaFlo \\
EAGLE EYE PLATINUM & Philips-Volcano & 20 & 20 & 0.014 " & Gray-scale, VH, ChromaFlo \\
OPTICROSS 18 & Boston Scientific & 30 & 12 & 0.018 " & Gray-scale \\
\hline
\end{tabular}


can be referred to a detailed review by Spacek et al on these imaging modalities. ${ }^{6}$

\section{Detection of calcium severity}

The presence of severe calcification increases the complexity of a procedure, coronary or peripheral, and yields a high rate of complications. Severe calcium interferes with adequate vessel expansion with balloon angioplasty or stenting. ${ }^{7}$ A 90 -degree arc of calcium seen on IVUS is considered mild whereas an arc of 180-270 degrees or more is moderate to severe. ${ }^{8}$ The presence of severe calcium lowers procedural success with likely more dissection and need for stenting. Also, it can prevent balloon crossing to the target lesion. ${ }^{9}$ Currently, the presence of severe calcium may require vessel prepping typically with atherectomy, lithoplasty or scoring devices. Identifying the degree of calcification may help the operator determine the best strategy to tackle these complex lesions.

Fanelli and colleagues noted that severe calcium defined by an arc of calcium of 270 degrees and more within the vessel interferes with the effectiveness of drugcoated balloons and lead to loss of patency (Figure 1). ${ }^{8}$ Similar findings were reported in the Definitive AR study that noted approximately similar reduction in patency with the presence of severe calcifications. ${ }^{10}$ Several studies in the coronaries also indicated that calcium can reduce procedural success and debulking of calcium can improve procedural success. ${ }^{11}$ Currently, the Eclipse trial is

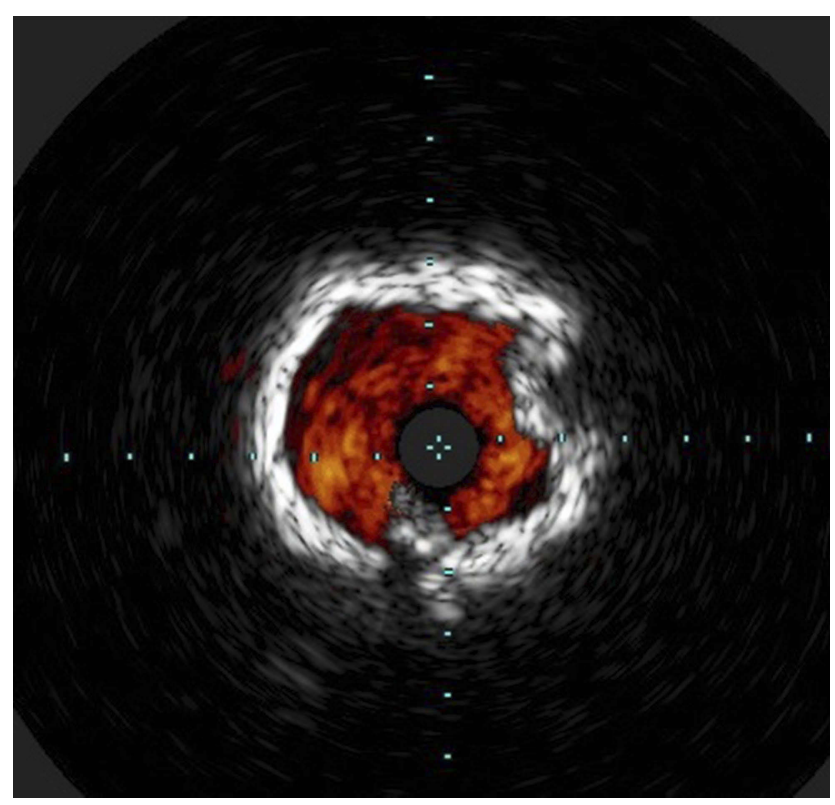

Figure I Severe 360-degree calcium. evaluating the value of adding orbital atherectomy to drug-eluting stents vs drug-eluting stents alone in treating complex calcified coronary lesions. ${ }^{12}$

Angiography underestimates the degree of calcification within a vessel. IVUS, on the other hand, has been shown to be more sensitive in detecting calcium in vessel walls as well its degree of severity. ${ }^{13,14}$ Mintz et al reported that calcium was seen in $38 \%$ of coronary lesions via angiography and $73 \%$ via IVUS, and calcium quite often is underappreciated in smaller vessels. ${ }^{13,14}$ Yin and colleagues noted that IVUS-detected calcium in $93.6 \%$ of peripheral arterial lesions whereas angiography-detected calcium in $55.3 \%$ of the same lesions. ${ }^{15}$ The positive predictive value and negative predictive value of angiography relative to IVUS were $100 \%$ and $14 \%$, respectively. Detecting the severity of calcium in a vessel may influence the strategy of treatment. The cost-effectiveness of routinely using IVUS in endovascular interventions needs to be proven in future clinical trials.

\section{Detection of thrombus}

Angioscopy is the gold standard for identifying thrombus. Studies indicated that angiography has a low sensitivity but a high specificity in identifying intravascular thrombus when compared to angioscopy. ${ }^{16}$ However, the availability of angioscopy in endovascular laboratories is limited. In the DETHROMBOSIS study that evaluated the role of power pulse spray using the AngioJet device combined with rheolytic therapy in treating subacute or recently occluded ( $<6$ months and $>24$ hrs) femoropopliteal lesions, 16/17 patients had thrombus identified by IVUS versus $7 / 17$ patients by angiography (Figure 2). ${ }^{17}$ Fresh, acute and non-occlusive thrombus may not be clearly visualized by IVUS however because of a high concentration of red blood cells and low fibrin deposition in these lesions. In the coronaries, IVUS also play an important role in defining hazy lesions that may be dissections, thrombus or recanalized chronic coronary thrombus. Thrombotic lesions may carry the potential for embolization. As of today, there are no embolic protection devices approved for native coronary arteries and routine aspiration of thrombus in the coronaries does not alter mortality and may increase the risk of embolic stroke. ${ }^{18,19,20}$

\section{Detection of dissections}

Dissections have been traditionally assessed and graded by angiography. The NHLBI classification has been the traditional grading system that has been widely utilized. 


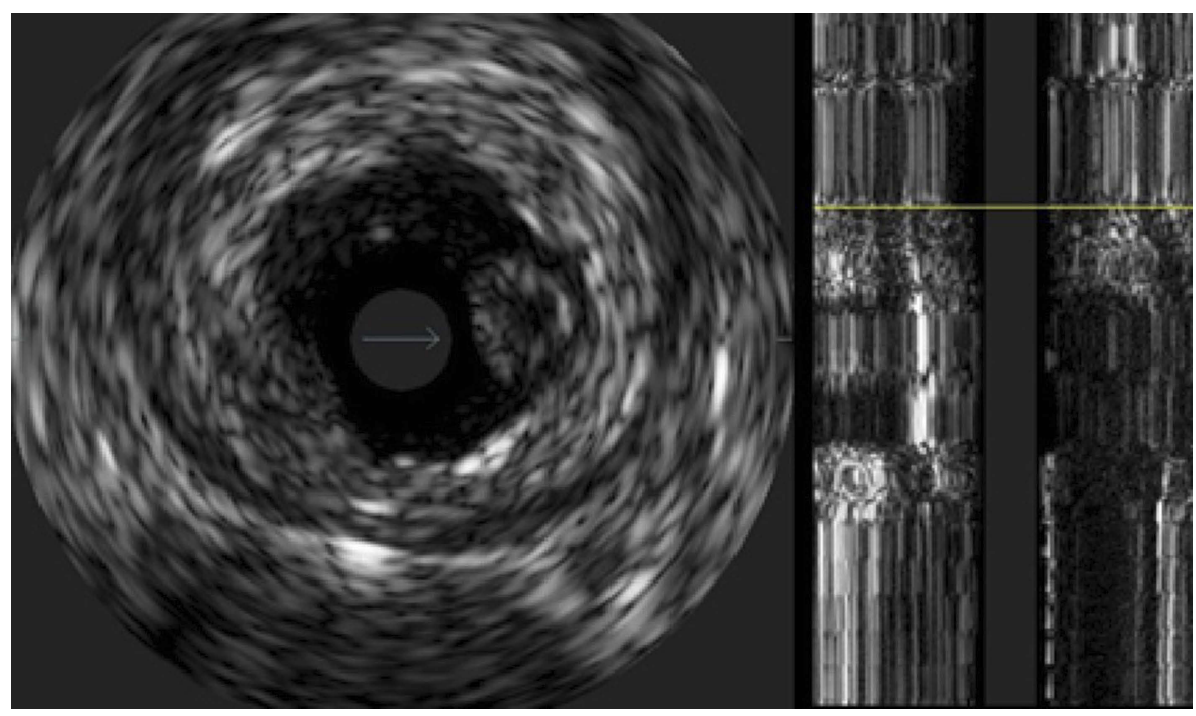

Figure 2 Thrombus.

Note: The arrow represents the thrombus.

Angiography was recently demonstrated to underestimate the number of dissections (Figure 3). Also, it has missed a large number of deeper dissections involving the media or adventitia as well as the arc of circumference of the dissection. The iDissection above the knee study showed, using core lab adjudicated IVUS and angiography, that
IVUS can identify 4-6 times more dissections than angiography. ${ }^{21}$ Also, by IVUS, $39 \%$ of dissections following Jetstream atherectomy involved the media and adventitia. The iDissection grading system combines both depth (Intima, media and adventitia) and circumference $(<180$ degrees and $\geq 180$ degrees) of dissections and therefore
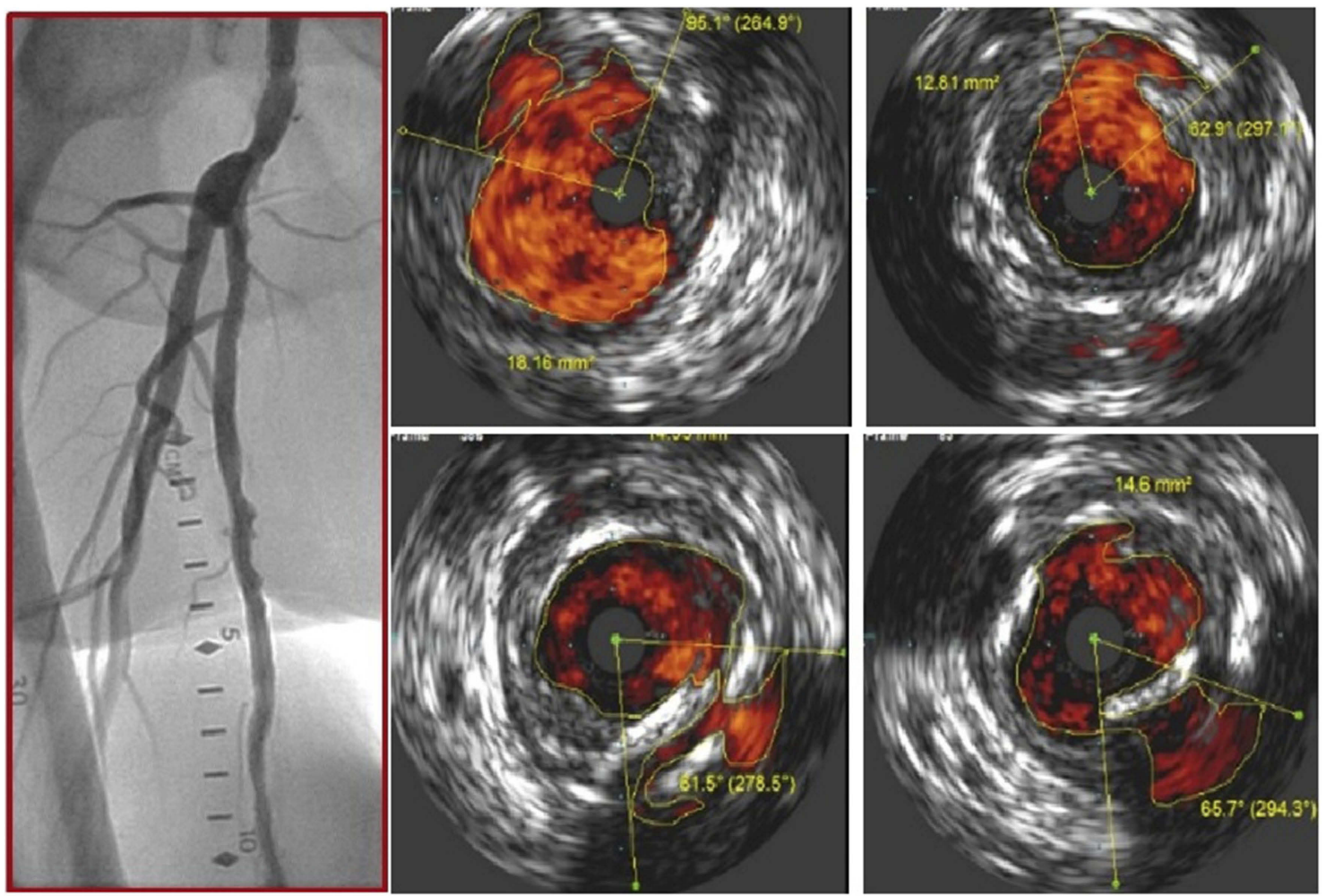

Figure 3 Multiple dissections seen on intravascular ultrasound that are not visible on angiogram. 
have the potential to predict long-term (patency) and shortterm outcomes (acute closure and bailout stenting), respectively. ${ }^{22}$ Deeper dissections are likely to trigger a cascade of restenosis whereas larger flaps are likely to fall within the lumen and cause acute or subacute thrombotic events or require adjunctive stenting. ${ }^{23}$ Data indicate that following angioplasty of the superficial femoral artery, the extent of dissections, free lumen area and diameter seen with IVUS are predictive factors of patency. ${ }^{24}$

In the coronaries, IVUS can identify dissections and intramural hematoma. IVUS can play an important role in identifying edge dissections which may lead to stent thrombosis. Also, IVUS can be useful in making the diagnosis in patients with spontaneous coronary artery dissections. Hazy intracoronary lesions can be dissections or thrombus and IVUS is an important tool to more accurately define these lesions. Finally, intramural hematoma identified on IVUS are associated with non-ST elevation myocardial infarction, sudden death and repeat revascularization. ${ }^{25}$

\section{Vessel diameter and lesion severity}

Vessel diameter and lesion severity are quite often underestimated on angiography. This is due in part to the diffuse nature of atherosclerosis and vessel tortuosity. Kashyab et al evaluated peripheral arterial angiography segments by histology. He noted a significantly greater linear stenosis when both internal elastic lamina (IEL) and external elastic lamina (EEL) were measured on histology specimens than angiography. The authors concluded that angiography underestimates true lumen size, and highlighted the importance of relying on other modalities to assess arterial lesion in peripheral arterial vascular beds. ${ }^{26}$ Similarly, Mintz et al studied 884 symptomatic coronary artery disease patients undergoing therapeutic and diagnostic coronary intervention with IVUS. They noted that only $6.8 \%$ of angiographically normal segments were normal on IVUS. ${ }^{13}$ Furthermore, angiography can undersize vessel size by as much as $\sim 30 \%$ when treating infrapopliteal disease, a problem that seems to become more noticeable in smaller vessels. $^{27,28}$

Underestimating vessel diameter may have significant implications on various therapeutic interventions. Undersized balloons may result in suboptimal vessel expansion and less than optimal luminal area gains. In the era of drug-coated balloons, this may result in a lack of apposition of the balloon to the vessel wall and therefore loss of optimal drug delivery to the tissue. Furthermore, undersized stents could lead to stent malapposition and increasing the risk of restenosis and late thrombosis. ${ }^{29}$

\section{Stent apposition and expansion}

Stenting of infrainguinal arteries have higher patency rates and reduced target lesion revascularization than angioplasty. Also, drug-eluting stents were shown to be superior to bare metal stents in treating lower extremity arterial vasculature yielding a reduced late lumen loss and restenosis rates. ${ }^{30-32}$ However, despite improvement in outcomes with stenting, the 1-year primary patency rate was approximately $80 \%$. The ACHILLES trial, a prospective randomized study, compared the in-segment binary restenosis rate in patients with infrapopopliteal vascular disease treated with sirolimus-eluting stents (SES) versus percutaneous transluminal balloon angioplasty (PTA) over a mean follow-up time of 1 year. ${ }^{33}$ The study included 200 patients, of which 99 were randomly assigned to the SES group and 101 to the PTA group. Results showed a lower restenosis rate and greater vessel patency in the SES-treated patients compared to the PTAtreated group $(22.4 \%$ vs $41.9 \%, P=0.019)$ and $(75.0 \%$ vs $57.1 \%, P=0.025)$, respectively. Similar results were also seen in patients with superficial femoral artery (SFA) disease. The IMPERIAL trial, the first head-to-head DES trial in the SFA, is a randomized single-blinded prospective study that compared the primary patency rates and major adverse events between Eluvia (a polymer-coated paclitaxel-eluting stent) and Zilver PTX (a polymer-free paclitaxel-coated stent) over a mean follow-up time of 1 year. ${ }^{34}$ A total of 465 patients were randomly assigned in a 2:1 ratio into two groups: Eluvia $(n=309)$ or Zilver PTX $(n=156)$. Results demonstrated non-inferiority between the two groups in respect to the primary endpoints; primary patency rates $(86.8 \%$ vs $81.5 \%, P<0.0001)$ and no major adverse events $(94.9 \%$ vs $91.0 \%, P<0.0001)$.

Stent under-expansion and incomplete apposition to the vessel wall can lead to the delivery of sub-therapeutic drug concentrations to the vessel wall and thus incomplete inhibition of smooth muscle cell proliferation. IVUSguided stenting has been shown to be superior to the conventional angiographic stenting in patients with coronary artery disease in terms of subacute stent thrombosis. ${ }^{35}$ Also, Waksman et al showed that IVUS-guided coronary bare metal stents reduced restenosis and the need for revascularization procedures but failed to demonstrate clinically significant difference in myocardial infarction or death. ${ }^{7}$ Similar results have also been demonstrated in 
PAD. In a small study of 49 Iliac segments that underwent stenting, Buckley et al showed a $100 \%$ primary patency rates in patients treated with IVUS guidance versus $82 \%$ in the non-IVUS patient group. ${ }^{36}$ This study also revealed that $20 / 49(41 \%)$ patients in the IVUS group had suboptimal stent deployment, despite evidence of adequate expansion and apposition by means of angiographic evaluation.

\section{Limitations of IVUS}

Although observational and retrospective studies indicate a high rate of patency and freedom from revascularization with the use of IVUS as an adjunctive imaging modality, data from randomized trials remain lacking. Assessment and optimization of stent deployment in coronary and peripheral artery disease and the identification of post-procedural dissections that are not easily picked up by angiography alone can be accomplished with the use of IVUS. ${ }^{21,35,36}$ A universal algorithm however for the use of IVUS prior to peripheral or coronary interventions has not been adopted to optimize long-term outcomes despite a notable significant improvement in acute procedural results and short-term outcomes. ${ }^{7,36,37}$ In addition, IVUS leads to a decrease in the amount of contrast used and its associated complications in PCI and peripheral vascular procedures but its cost-effectiveness has not been conclusively demonstrated. A cost-effective analysis by Panaich et al from a nationwide inpatient data sample on the use of IVUS in lower limb interventions showed that the cost of using IVUS was offset by lower complications in the IVUS group. ${ }^{38}$ However, other studies indicate that IVUS can add a significant cost to a procedure. ${ }^{39}$ Furthermore, IVUS requires a level of expertise in image interpretation that may not be available in all centers. This hinders a wide adoption of this technology. The current pairing of IVUS with angiographic imaging using SyncVision (Philips) offers an easy tool to match IVUS findings on an angiogram. This functionality is however not available for peripheral vascular imaging.

\section{Practical applications of IVUS in our practice}

The use of IVUS in the periphery to assess plaque morphology has allowed us to identify large soft plaques with lipid core, ulcerated/necrotic plaques or presence of thrombus that are likely to embolize with percutaneous interventions, lowering the threshold for the use of distal embolic protection devices. Also, the choice of the vessel prepping device has been directed by the nature of the visualized plaque.
Severely calcified plaques are treated with atherectomy prior to more definitive treatment to alter vessel compliance, reduce dissections and bailout stenting and improve the efficacy of drug elution devices. In addition, dissections seen on IVUS and not clearly identified on angiogram are treated aggressively with the Tack endovascular system or spot stenting if they are of a large arc (>180 degrees) or deep reaching the media/adventitia to enhance the acute and possibly long-term results of the procedure. Furthermore, assessing the acute procedural results and obtaining the best minimal luminal gain or stent expansion have been also assessed with IVUS. Finally, the choice of balloon size (particularly important with drug-coated balloons) and full lesion coverage are more effectively guided by IVUS. It should be noted that these IVUS applications will need further validation for cost-effectiveness.

\section{Conclusion}

Angiography remains an important and practical way to evaluate the vasculature. However, it has several limitations. Adjunctive imaging modalities such as IVUS can provide a more accurate visualization to what is happening within the blood vessel. Functional testing offers an excellent way to assess significance of moderate coronary lesions but its applicability in peripheral interventions remains limited. IVUS is easy to use and may be widely available but requires expertise in image interpretation which may limit its routine use. ${ }^{39}$

\section{Disclosure}

Dr Shammas receives educational and research grants from Boston Scientific, Intact Vascular, Bard, VentureMed Group, Phillips and is on the speaker bureau of Janssen, Boehringer Ingelheim, Novartis and Zoll Medical. The authors report no other conflicts of interest in this work.

\section{References}

1. Kruger RA, Mistretta CA, Crummy AB, et al. Digital K-edge subtraction radiography. Radiology. 1977;125(1):243-245. doi:10.1148/125.1.243

2. Arthurs ZM. The evaluation of peripheral vascular disease with intrascular ultrasound. Vasc Dis Manag. 2011;8(4):E81-E86.

3. Mintz GS, Nissen SE, Anderson WD, et al. American College of Cardiology clinical expert consensus document on standards for acquisition, measurement and reporting of intravascular ultrasound studies (IVUS). A report of the American College of Cardiology task force on clinical expert consensus documents. J Am Coll Cardiol. 2001;37 (5):1478-1492. doi:10.1016/s0735-1097(01)01175-5

4. Garcìa-Garcìa HM, Gogas BD, Serruys PW, Bruining N. IVUS-based imaging modalities for tissue characterization: similarities and differences. Int J Cardiovasc Imaging. 2011;27:215-224. doi:10.1007/s10554-0109789-7 
5. Stone GW, Maehara A, Lansky AJ, et al. A prospective naturalhistory study of coronary atherosclerosis. N Engl J Med. 2011;364 (3):226-235. doi:10.1056/NEJMoa1002358

6. Spacek M, Zemanek D, Hutyra M, Sluka M, Taborsky M. Vulnerable atherosclerotic plaque - a review of current concepts and advanced imaging. Biomed Pap Med Fac Univ Palacky Olomouc Czech Repub. 2018;162:10-17. doi:10.5507/bp.2018.004

7. Waksman R, Kitabata H, Prati F, Albertucci M, Mintz GS. Intravascular ultrasound versus optical coherence tomography guidance. J Am Coll Cardiol. 2013;62(17 Suppl):S32-S40. doi:10.1016/ j.jacc.2013.08.709

8. Fanelli F, Cannavale A, Gazzetti M, et al. Calcium burden assessment and impact on drug-eluting balloons in peripheral arterial disease. Cardiovasc Intervent Radiol. 2014;37(4):898-907. doi:10.1007/s00270-014-0904-3

9. Rocha-Singh KJ, Zeller T, Jaff MR. Peripheral arterial calcification: prevalence, mechanism, detection, and clinical implications. Catheter Cardiovasc Interv. 2014;83(6):E212-E220. doi:10.1002/ccd.25387

10. Zeller T, Langhoff R, Rocha-Singh KJ, et al.; DEFINITIVE AR Investigators. Directional atherectomy followed by a paclitaxelcoated balloon to inhibit restenosis and maintain vessel patency: twelve-month results of the DEFINITIVE AR study. Circ Cardiovasc Interv. 2017;10(9):pii: e004848. doi:10.1161/ CIRCINTERVENTIONS.116.004848

11. Lee M, Généreux P, Shlofmitz R, et al. Orbital atherectomy for treating de novo, severely calcified coronary lesions: 3-year results of the pivotal ORBIT II trial. Cardiovasc Revasc Med. 2017;18:261264. doi:10.1016/j.carrev.2017.01.011

12. Evaluation of treatment strategies for severe CaLcIfic coronary arteries: orbital atherectomy vs. conventional angioplasty technique prior to implantation of drug-eluting StEnts: the ECLIPSE trial (ECLIPSE). Available from: http://www.Clinicaltrials.gov. NLM identifier: NCT03108456. Accessed March 28, 2019.

13. Mintz GS, Popma JJ, Pichard AD, et al. Patterns of calcification in coronary artery disease. A statistical analysis of intravascular ultrasound and coronary angiography in 1155 lesions. Circulation. 1995;91(7):1959-1965. doi:10.1161/01.cir.91.7.1959

14. Mintz GS, Pichard AD, Kent KM, Satler LF, Popma JJ, Leon MB. Interrelation of coronary angiographic reference lumen size and intravascular ultrasound target lesion calcium. Am J Cardiol. 1998;81(4):387-391. doi:10.1016/s0002-9149(97)00924-7

15. Yin D, Maehara A, Shimshak TM, et al. Intravascular ultrasound validation of contemporary angiographic scores evaluating the severity of calcification in peripheral arteries. J Endovasc Ther. 2017;24:478-487.

16. Den Heijer P, Foley DP, Escaned J, et al. Angioscopic versus angiographic detection of intimal dissection and intracoronary thrombus. $\mathrm{J}$ Am Coll Cardiol. 1994;24:649-654. doi:10.1016/0735-1097(94)90010-8

17. Shammas NW, Dippel EJ, Shammas G, Gayton L, Coiner D, Jerin M. Dethrombosis of the lower extremity arteries using the power-pulse spray technique in patients with recent onset thrombotic occlusions results of the DETHROMBOSIS registry. $J$ Endovasc Ther. 2008;15:570-579. doi:10.1583/08-2453.1

18. Jolly SS, James S, Džavík V, et al. Thrombus aspiration in STsegment-elevation myocardial infarction: an individual patient metaanalysis: thrombectomy trialists collaboration. Circulation. 2017;135:143-152. doi:10.1161/CIRCULATIONAHA.116.025371

19. Mastoris I, Giustino G, Sartori S, et al. Efficacy and safety of routine thrombus aspiration in patients with ST-segment elevation myocardial infarction undergoing primary percutaneous coronary intervention: an updated systematic review and meta-analysis of randomized controlled trials. Catheter Cardiovasc Interv. 2016;87:650-60.16c. doi: $10.1002 / \mathrm{ccd} .26152$

20. Aghlmandi S, Schärer N, Heg D, et al. Thrombus aspiration in acute coronary syndromes: prevalence, procedural success, change in serial troponin $\mathrm{T}$ levels and clinical outcomes in a contemporary Swiss cohort. Eur Heart J Acute Cardiovasc Care. 2018;7:522-531. doi:10.1177/2048872617706480
21. Shammas NW, Torey JT, Shammas WJ, Jones-Miller S, Shammas GA. Intravascular ultrasound assessment and correlation with angiographic findings demonstrating femoropopliteal arterial dissections post atherectomy: results from the iDissection study. J Invasive Cardiol. 2018;30:240-244.

22. Shammas NW, Torey JT, Shammas WJ. Dissections in peripheral vascular interventions: a proposed classification using intravascular ultrasound. J Invasive Cardiol. 2018;30(4):145-146.

23. Tarricone A, Ali Z, Rajamanickam A, et al. Histopathological evidence of adventitial or medial injury is a strong predictor of restenosis during directional atherectomy for peripheral artery disease. $J$ Endovasc Ther. 2015;22:712-715. doi:10.1177/1526602815597683

24. Gussenhoven EJ, van der Lugt A, Pasterkamp G, et al. Intravascular ultrasound predictors of outcome after peripheral balloon angioplasty. Eur J Vasc Endovasc Surg. 1995;10:279-288.

25. Maehara A, Mintz GS, Bui AB, et al. Incidence, morphology, angiographic findings, and outcomes of intramural hematomas after percutaneous coronary interventions: an intravascular ultrasound study. Circulation. 2002;105:2037-2042. doi:10.1161/01. cir.0000015503.04751.bd

26. Kashyap VS, Pavkov ML, Bishop PD, et al. Angiography underestimates peripheral atherosclerosis: lumenography revisited. $J$ Endovasc Ther. 2008;15:117-125. doi:10.1583/07-2249R.1

27. Shammas NW, Shammas WJ, Armstrong EJ, Radaideh Q, Shammas GA. Are we appropriately treating infrapopliteal arterial disease? The need for precision imaging with intravascular ultrasound. Vasc Dis Manag. 2018;15(12):E137-E139.

28. Briguori C, Tobis J, Nishida T, et al. Discrepancy between angiography and intravascular ultrasound when analysing small coronary arteries. Eur Heart J. 2002;23:247-254. doi:10.1053/euhj.2001.2730

29. Fuentes L, Gómez-Lara J, Salvatella N, et al. IVUS findings in late and very late stent thrombosis. A comparison between bare-metal and drug-eluting stents. Rev Esp Cardiol (Engl Ed). 2018;71:335-343. doi:10.1016/j.rec.2017.08.001

30. Laird JR, Hong M. Drug-eluting stents in the superficial femoral artery: the long and winding road. Circulation. 2016;133:14351437. doi:10.1161/CIRCULATIONAHA.116.022039

31. Rastan A, Tepe G, Krankenberg H, et al. Sirolimus-eluting stents vs. bare-metal stents for treatment of focal lesions in infrapopliteal arteries: a double-blind, multi-centre, randomized clinical trial. Eur Heart J. 2011;32(18):2274-2281. doi:10.1093/eurheartj/ehr144

32. Bosiers M, Scheinert D, Peeters P, et al. Randomized comparison of everolimus-eluting versus bare-metal stents in patients with critical limb ischemia and infrapopliteal arterial occlusive disease. $J$ Vasc Surg. 2012;55(2):390-398. doi:10.1016/j.jvs.2011.07.099

33. Scheinert D, Katsanos K, Zeller T, et al. A prospective randomized multicenter comparison of balloon angioplasty and infrapopliteal stenting with the sirolimus-eluting stent in patients with ischemic peripheral arterial disease: 1-year results from the ACHILLES trial. $J$ Am Coll Cardiol. 2012;60:2290-2295. doi:10.1016/j.jacc.2012.08.989

34. Gray WA, Keirse K, Soga Y, et al. A polymer-coated, paclitaxeleluting stent (Eluvia) versus a polymer-free, paclitaxel-coated sten (Zilver PTX) for endovascular femoropopliteal intervention (IMPERIAL): a randomised, non-inferiority trial. Lancet. 2018;392 (10157):1541-1551. doi:10.1016/S0140-6736(18)32262-1

35. Bonello L, De Labriolle A, Lemesle G, et al. Intravascular ultrasound-guided percutaneous coronary interventions in contemporary practice. Arch Cardiovasc Dis. 2009;102(2):143-151. doi:10.1016/j. acvd.2008.11.002

36. Buckley CJ, Arko FR, Lee S, et al. Intravascular ultrasound scanning improves long-term patency of iliac lesions treated with balloon angioplasty and primary stenting. J Vasc Surg. 2002;35(2):316-323.

37. Iida O, Takahara M, Soga Y, et al. Efficacy of intravascular ultrasound in femoropopliteal stenting for peripheral artery disease with TASC II class A to C lesions. J Endovasc Ther. 2014;21(4):485-492. doi:10.1583/14-4721R.1 
38. Panaich SS, Arora S, Patel N, et al. Intravascular ultrasound in lower extremity peripheral vascular interventions: variation in utilization and impact on in-hospital outcomes from the nationwide inpatient sample (2006-2011). J Endovasc Ther. 2016;23:65-75. doi:10.1177/ 1526602815620780
39. Makris GC, Chrysafi P, Little M, et al. The role of intravascular ultrasound in lower limb revascularization in patients with peripheral arterial disease. Int Angiol. 2017;36(6):505-516. doi:10.23736/ S0392-9590.17.03866-4

\section{Publish your work in this journal}

Vascular Health and Risk Management is an international, peerreviewed journal of therapeutics and risk management, focusing on concise rapid reporting of clinical studies on the processes involved in the maintenance of vascular health; the monitoring, prevention and treatment of vascular disease and its sequelae; and the involvement of metabolic disorders, particularly diabetes. This journal is indexed on PubMed Central and MedLine. The manuscript management system is completely online and includes a very quick and fair peerreview system, which is all easy to use. Visit http://www.dovepress com/testimonials.php to read real quotes from published authors. 